[4] M. G. Grigorian, On some properties of orthogonal systems, Izv. Ross. Akad. Nauk Ser. Mat. 57 (1993), no. 5, 75-105.

[5] N. N. Luzin, On the fundamental theorem of the integral calculus, Mat. Sb. 28 (1912), 266-294 (in Russian).

[6] D. E. Men'shov, On Fourier series of integrable functions, Trudy Moskov. Mat. Obshch. 1 (1952), 5 -38.

Department of Physics

State University of Yerevan

Alek Manukian 1

375019 Yerevan, Republic of Armenia.

E-mail: instmath@sci.am

\section{The Conley index in Hilbert spaces and its applications}

by

K. GEBA, M. IZYDOREK and A. PRUSZKO (Sopot)

Abstract. We present a generalization of the classical Conley index defined for flows on locally compact spaces to flows on an infinite-dimensional real Hilbert space $H$ generated by vector fields of the form $f: H \rightarrow H, f(x)=L x+K(x)$, where $L: H \rightarrow H$ is a bounded linear operator satisfying some technical assumptions and $K$ is a completely continuous perturbation. Simple examples are presented to show how this new invariant can be applied in searching critical points of strongly indefinite functionals having asymptotically linear gradient.

1. Introduction. The purpose of this paper is to present a new generalization of the classical Conley index theory. The standard reference for that theory, developed by Charles Conley in the 70's, is his monograph Isolated Invariant Sets and the Morse Index [5]. Referring for all technical details to [5] and the recent paper of Mischaikow [12], we recall that in Conley index theory, with any compact isolated invariant set $S$ of a flow $\eta: \mathbb{R} \times Z \rightarrow Z$ on a locally compact metric space $Z$ one can associate an index $h(S)$, which is the homotopy type of a compact pointed space. Instead of isolated invariant sets one can equivalently consider (compact) isolating neighbourhoods for flows as pointed out by Mischaikow [12]. Since our construction of Conley index is analogous to the construction of the Leray-Schauder degree it seems more convenient to work with isolating neighbourhoods than directly with isolated invariant sets.

The aim of this paper is to extend the ideas of Conley to the case where $Z$ is replaced by an infinite-dimensional Hilbert space. To be more precise, we assume that we are given an infinite-dimensional real Hilbert space $H$ together with a bounded linear operator $L: H \rightarrow H$ such that $H$ and $L$ satisfy conditions (H.1), (H.2) and (H.3) below. We will be concerned with local flows on $H$ generated by $\mathcal{L} \mathcal{S}$-vector fields, i.e. maps $f: H \rightarrow H$ which can be written in the form $f(x)=L x+K(x)$, where $K: H \rightarrow H$ is a suff-

1991 Mathematics Subject Classification: Primary 58E05, 34C35; Secondary 34C25.

Research of K. Gęba supported by KBN grant 2-PO3A-08008.

Research of A. Pruszko supported by KBN grant 0008/PO3/9611. 
ciently smooth, completely continuous mapping. This kind of flows appear, for instance, when one applies variational methods to prove the existence or multiplicity results for periodic solutions of some types of Hamiltonian systems (see [1], [6], [17], [3] and the references given there), second order ODE's (see [2], [11] and the references given there) as well as of some elliptic. and hyperbolic problems (see [1], [4], [13], [14], [18]). Our index inherits all basic properties of the classical one, in particular, it remains invariant under "small" perturbations.

As a starting point we take a well known fact- the similarities shared by degree theory and the Conley index. Our construction generalizes the classical Conley index in precisely the same way as the Leray Schauder degree generalizes the topological (Brouwer) degree.

Actually, writing this article we were motivated by A. Szulkin's papers [17] and [18] in which many different index theories of Morse type with applications to strongly indefinite functionals have been described. A fundamental paper concerning that subject is $\mathrm{H}$. Amann and $\mathrm{E}$. Zehnder [1]. Morse theory in Hilbert spaces is thoroughly discussed in [3] and [11]. One should also mention $K$. Rybakowski's book [15] in which a version of the Conley index for flows in non-locally compact spaces is defined. Applications to nonlinear elliptic and parabolic equations are also presented. However, it seems that Rybakowski's index cannot be applied to strongly indefinite functionals.

The paper is organized as follows. In Section 2 we present basic definitions and facts needed to develop our theory. The crucial result is Proposition 2.3 which says that if $\eta$ is an $\mathcal{L S}$-flow on $H$ then any bounded isolated invariant set $S$ for $\eta$ is compact. Section 3 is devoted to the notion of spectrum well known to algebraic topologists. Spectra are discussed in detail by G. W. Whitehead [20]. However, the purpose of using spectra in our paper is completely different. Consequently, the main definitions are modified in order to make them applicable to our considerations. In Section 4 the construction of the index is presented together with its basic properties: "nontriviality" (Proposition 4.4) and "continuation" (Proposition 4.5). Finally, in Section 5 we give a few examples to show how our theory can be applied in searching critical points of strongly indefinite functionals which are asymptotically quadratic. We point out that our examples are not covered by the theorems we have found in the literature for that kind of problems.

2. Isolating neighbourhoods. Throughout this section we assume that $H=(H,\langle\cdot, \cdot\rangle)$ is a real Hilbert space and $L: H \rightarrow H$ is a linear bounded operator with spectrum $\sigma(L)$ such that:

(H.1) $\quad H=\bigoplus_{k=0}^{\infty} H_{k}$ with all subspaces $H_{k}$ being mutually orthogonal and of finite dimension;
(H.2) $\quad L\left(H_{0}\right) \subset H_{0}, H_{0}$ is the invariant subspace of $L$ corresponding to the part $\sigma_{0}(L):=i \mathbb{R} \cap \sigma(L)$ of the spectrum lying on the imaginary axis and $L\left(H_{k}\right)=H_{k}$ for all $k>0$;

$\left(\right.$ H.3) $\quad \sigma_{0}(L)$ is isolated in $\sigma(L)$, i.e. $\sigma_{0}(L) \cap \mathrm{cl}\left(\sigma(L) \backslash \sigma_{0}(L)\right)=\emptyset$.

Throughout the section $\Lambda$ denotes a compact metric space.

Recall that a continuos map $\eta: D(\eta) \rightarrow H$ is a local flow on $H$ if

(F.1) $D(\eta)$ is an open subset of $\mathbb{R} \times H$ such that $\{0\} \times H \subset D(\eta)$;

(F.2) for every $x \in H$ there exist $\alpha(x), \omega(x) \in \mathbb{R} \cup\{-\infty, \infty\}$ such that $(\alpha(x), \omega(x))=\{t \in \mathbb{R}:(t, x) \in D(\eta)\}$

(F.3) $\quad \eta(t, \eta(s, x))=\eta(t+s, x)$ for all $x \in H$ and $t, s \in(\alpha(x), \omega(x))$ such that $t+s \in(\alpha(x), \omega(x))$.

A local flow $\eta$ is a flow on $H$ if $D(\eta)=\mathbb{R} \times H$.

If $\eta$ is a local flow on $H$ and $X \subset H$ then

$\operatorname{lnv}(X)=\operatorname{Inv}(X, \eta):=\{x \in X: \eta(t, x) \in X$ for all $t \in(\alpha(x), \omega(x))\}$

is the maximal $\eta$-invariant subset of $X$.

We say that a continuous map $\eta: D(\eta) \rightarrow H$ is a family of local flows on $H$ indexed by $\Lambda$ if $D(\eta)$ is an open subset of $\mathbb{R} \times H \times \Lambda$ and $\eta_{\lambda}$ (where $\left.\eta_{\lambda}(t, x)=\eta(t, x, \lambda)\right)$ is a local flow for every $\lambda \in \Lambda$. If $D(\eta)=\mathbb{R} \times H \times \Lambda$ then we say that $\eta$ is a family of flows on $H$ indexed by $\Lambda$.

If $\eta$ is a family of local flows indexed by $A$ on $X \subset H$ then we let

$$
\operatorname{Inv}(X, \eta):=\left\{(x, \lambda) \in X \times \Lambda: x \in \operatorname{Inv}\left(X, \eta_{\lambda}\right)\right\} .
$$

We say that $F: H \times \Lambda \rightarrow H$ (resp. $F: \mathbb{R} \times H \times \Lambda \rightarrow H$ ) is a completely continuous map if $F$ is continuous and for any bounded subset $A \subset H$ (resp., $A \subset \mathbb{R} \times H)$ the closure of $F(A \times \Lambda)$ is a compact subset of $H$.

DEFINITION 2.1. We say that a flow $\eta: \mathbb{R} \times H \rightarrow H$ is an $\mathcal{L}$-flow if

$$
\eta(t, x)=e^{t L} x+U(t, x)
$$

where $U: \mathbb{R} \times H \rightarrow H$ is completely continuous.

DEFINITION 2.2. Let $\Lambda$ be a compact metric space. We say that a family of flows $\eta: \mathbb{R} \times H \times \Lambda \rightarrow H$ is a family of $\mathcal{L} \mathcal{S}$-flows if

$$
\eta(t, x, \lambda)=e^{t L} x+U(t, x, \lambda)
$$

where $U: \mathbb{R} \times H \times \Lambda \rightarrow H$ is completely continuous.

Proposimion 2.3. Let $\Lambda$ be a compact metric space and let $\eta: \mathbb{R} \times H \times$ $\Lambda \rightarrow H$ be a family of $\mathcal{L}$-flows. If $X \subset H$ is closed and bounded then $S:=\operatorname{Inv}(X \times \Lambda, \eta)$ is a compact subset of $X \times \Lambda$.

Proof. Let $H_{-}$(resp. $H_{+}$) denote the invariant subspace of $L$ corresponding to the eigenvalues with negative (resp. positive) real part. Clearly, $H$ splits into the direct sum $H_{-} \oplus H_{0} \oplus H_{+}$. 
Let $P_{-}\left(\right.$resp. $\left.P_{0}, P_{+}\right)$denote the orthogonal projection of $H$ onto $H$ (resp. $\left.H_{0}, H_{+}\right)$. Since $\sigma_{0}(L)$ is isolated in $\sigma(L)$, it follows that for every $\varrho>0$ there is $T_{0} \in(0, \infty)$ such that

$$
\begin{array}{ll}
\left\|e^{t L} x\right\| \geq \varrho\|x\| & \text { for all } t \geq T_{0}, x \in H_{+}, \\
\left\|e^{t L} x\right\| \geq \varrho\|x\| & \text { for all } t \leq-T_{0}, x \in H_{-} .
\end{array}
$$

It follows from the definition of $S$ that it is a closed subset of $H \times \Lambda$. Suppose that $S$ is not compact.

Let $S_{0}:=\{x \in X:(x, \lambda) \in S$ for some $\lambda \in \Lambda\}$. Obviously, $S_{0} \subset$ $\operatorname{cl}\left(P_{-}\left(S_{0}\right)\right) \times \operatorname{cl}\left(P_{0}\left(S_{0}\right)\right) \times \operatorname{cl}\left(P_{+}\left(S_{0}\right)\right)$. The set $\operatorname{cl}\left(P_{0}\left(S_{0}\right)\right)$ is compact as it is a closed and bounded subset of a finite-dimensional vector space. Therefore $\operatorname{cl}\left(P_{-}\left(S_{0}\right)\right)$ and $\mathrm{cl}\left(P_{+}\left(S_{0}\right)\right)$ cannot be compact simultaneously. With no loss of generality we may assume that $P_{+}\left(S_{0}\right)$ is not relatively compact. As $H_{+}$ is a complete metric space, there is $\varepsilon>0$ such that $P_{+}\left(S_{0}\right)$ does not admit a finite $\varepsilon$-net. Hence, one can choose a sequence $\left(x_{k}\right) \subset P_{+}\left(S_{0}\right)\left(x_{k}=P_{+}\left(y_{k}\right) ;\right.$ $\left.\left(y_{k}, \lambda_{k}\right) \in S\right)$ such that $\left\|x_{i}-x_{j}\right\| \geq \varepsilon$ whenever $i \neq j$. Choose $s \in \mathbb{R}$ such that $X \subset B(0, s)$ and $T \in \mathbb{R}$ so large that the inequality (2.1) is satisfied for $\varrho=3 s / \varepsilon$ whenever $t \geq T$. Set $u_{k}:=e^{T L} y_{k}$ and $v_{k}:=U\left(T, y_{k}, \lambda_{k}\right)$. Then

$$
\eta\left(T, y_{k}, \lambda_{k}\right)=e^{T L} y_{k}+U\left(T, y_{k}, \lambda_{k}\right)=u_{k}+v_{k} \in X \subset B(0, s) .
$$

Thus

$$
3 s \leq\left\|u_{i}-u_{j}\right\| \leq\left\|u_{i}+v_{i}\right\|+\left\|v_{i}-v_{j}\right\|+\left\|u_{j}+v_{j}\right\|<2 s+\left\|v_{i}-v_{j}\right\|
$$

so that

$$
\left\|v_{i}-v_{j}\right\|>s \quad \text { whenever } i \neq j .
$$

Since $v_{k} \in U(\{T\} \times S)$ and $U(\{T\} \times S)$ is relatively compact we have a contradiction.

Definition 2.4. We say that $f: H \rightarrow H$ is an $\mathcal{L}$-vector field if there exists a completely continuous and locally Lipschitz continuous map $K$ : $H \rightarrow H$ such that

$$
f(x)=L x+K(x) \quad \text { for all } x \in H .
$$

Definition 2.5. We say that $f: H \times A \rightarrow H$ is a family of $\mathcal{L S}$ S vector fields if there exists a completely continuous and locally Lipschitz continuous map $K: H \times \Lambda \rightarrow H$ such that

$$
f(x, \lambda)=L x+K(x, \lambda) \quad \text { for all }(x, \lambda) \in H \times \Lambda .
$$

If $f: H \rightarrow H$ is an $\mathcal{L S}$-vector field and $x \in H$ then it is well known (see e.g. [7]) that there exists the maximal $C^{1}$-curve

$$
(\alpha(x), \omega(x)) \ni t \mapsto \eta(t, x) \in H
$$

satisfying

$$
\frac{d \eta}{d t}=f \circ \eta, \quad \eta(0, x)=x
$$

Moreover, if we set

$$
D(\eta):=\{(t, x) \in \mathbb{R} \times H: \alpha(x)<t<\omega(x)\},
$$

then $D(\eta) \subset \mathbb{R} \times H$ is open and $\eta: D(\eta) \ni(t, x) \mapsto \eta(t, x) \in H$ is a local flow. In what follows we call $\eta$ the local flow induced by $f$.

Let $f(x)=L x+K(x)$ be an $\mathcal{L S}$-vector field. Then the local flow $\eta$ generated by $f$ can be written in the form

$$
\eta(t, x)=e^{t L} x+U(t, x)
$$

where $U: D(\eta) \rightarrow H$ is completely continuous (see [13]).

We say that $f$ is subquadratic if there exist $a, b>0$ such that

$$
|\langle K(x), x\rangle| \leq a\|x\|^{2}+b \quad \forall x \in H
$$

The following result is a direct consequence of Theorem 2.1 of [19]

Proposirion 2.6. Let $f(x)=L x+K(x)$ denote an $\mathcal{L S}$-vector field and let $\eta$ be the local flow generated by $f$. Then

(a) if $x \in H$ and $\left(t_{n}\right)$ is a sequence in $(\alpha(x), \omega(x))$ convergent either to $\alpha(x)$ or to $\omega(x)$ then $\left(\eta\left(t_{n}, x\right)\right)$ is an unbounded sequence (in $\left.H\right)$;

(b) if $f$ satisfies (2.3) then $f$ generates an $\mathcal{L S}$-flow.

Definition 2.7. Let $\eta: D(\eta) \rightarrow H$ be a local flow on $H$. We say that a bounded and closed subset $X \subset H$ is an isolating neighbourhood for $\eta$ if

$$
\operatorname{Inv}(X) \subset \operatorname{Int}(X) .
$$

THEOREM 2.8. Let $\Lambda$ be a compact metric space and let $\eta: \mathbb{R} \times H \times \Lambda$ $\rightarrow H$ be a family of $\mathcal{L S}$-flows. Assume that $X \subset H$ is an isolating neighbourhood for a flow $\eta_{\lambda_{0}}$ for some $\lambda_{0} \in \Lambda$. Then there is an open neighbourhood $V \subset \Lambda$ of $\lambda_{0}$ such that $X$ is an isolating neighbourhood for any flow $\eta_{\lambda}$ whenever $\lambda \in V$.

Pro of. Proposition 2.3 implies that $\operatorname{Inv}(X \times \Lambda, \eta)$ is a compact subset of $X \times \Lambda$. Since $\operatorname{Inv}(X \times \Lambda, \eta) \cap\left(X \times\left\{\lambda_{0}\right\}\right) \subset \operatorname{Int}(X)$ there exists an open neighbourhood $V$ of $\lambda_{0}$ in $\Lambda$ such that $\operatorname{Inv}(X \times \Lambda) \cap(X \times V) \subset \operatorname{Int}(X) \times V$. Therefore $\operatorname{Inv}\left(X, \eta_{\lambda}\right) \subset \operatorname{Int}(X)$ for all $\lambda \in V$.

Clearly the above theorem can be reformulated in the following way:

COROLlaRY 2.9. For any closed and bounded set $X \subset H$ the set

$$
\Lambda(X):=\left\{\lambda \in \Lambda: X \text { is an isolating neighbourhood for } \eta_{\lambda} \text { in } H\right\}
$$

is open in $\Lambda$. 
3. Spectra. Let $\mathcal{M}_{0}$ be the category of compact metrizable spaces with a base point. If $\left(X, x_{0}\right)$ and $\left(Y, y_{0}\right)$ are objects in $\mathcal{M}_{0}$ then the set of morphisms $\operatorname{Mor}(X, Y)$ consists of all continuous maps $f: X \rightarrow Y$ preserving the base points. Let $A$ be a closed subset of a compact metrizable space $X$. Then the quotient space $X / A$ is obtained from $X$ by collapsing $A$ to a point, the base point of $X / A ; X / A$ is an object of $\mathcal{M}_{0}$. If $X$ and $Y$ are objects in $\mathcal{M}_{0}$ (with base points $x_{0}$ and $y_{0}$ resp.) then the cartesian product $X \times Y$ is also an object in $\mathcal{M}_{0}$ (with base point $\left(x_{0}, y_{0}\right)$ ).

Denote by $I$ the unit interval with base point $\{0\}$, by $\partial I=S^{0}$ the subspace $\{0,1\}$ of $I$, and let $S=\left(S^{1}, s_{0}\right):=I / \partial I$. The suspension of a space $\left(X, x_{0}\right) \in \mathcal{M}_{0}$ is defined as the quotient space $(S X, *):=\left(S^{1} \times X\right) /\left(S^{1} \times\right.$ $\left.\left\{x_{0}\right\} \cup\left\{s_{0}\right\} \times X\right)$. In fact, the suspension is a functor in $\mathcal{M}_{0}$, which means in particular that for any map $f:\left(X, x_{0}\right) \rightarrow\left(Y, y_{0}\right)$ the suspension map $S f:(S X, * X) \rightarrow(S Y, * Y)$ is a morphism in that category. For any $m \in \mathbb{N}$ we define $S^{m} X=S\left(S^{m-1} X\right)$.

Finally, recall that $f \in \operatorname{Mor}(X, Y)$ is a homotopy equivalence if there is $g \in \operatorname{Mor}(Y, X)$ such that $g \circ f$ is homotopic to id $X$ and $f \circ g$ is homotopic to id $Y$, both homotopies relative base points. If $f: X \rightarrow Y$ is a homotopy equivalence then we say that the spaces $X$ and $Y$ are homotopy equivalent or have the same homotopy type. A standard reference for this section is Whitehead [20].

We are now in a position to define a category of spectra. Let $\nu: \mathbb{N} \rightarrow \mathbb{N}$ $\mathbb{N}=\{0,1,2, \ldots\}$, be a fixed map. We begin with the folowing

DEFINITION 3.1. A pair of sequences $E=\left(\left(E_{n}\right)_{n=n(E)}^{\infty},\left(\varepsilon_{n}\right)_{n=n(E)}^{\infty}\right)$ will be called a spectrum if the following conditions are satisfied:

(a) $\forall n \geq n(E), E_{n} \in \mathcal{M}_{0}$;

(b) $\forall n \geq n(E), \varepsilon_{n} \in \operatorname{Mor}\left(S^{\nu(n)} E_{n}, E_{n+1}\right)$;

(c) $\exists n_{1} \geq n(E), \forall n \geq n_{1}, \varepsilon_{n}$ is a homotopy equivalence.

DeFinition 3.2. A map of spectra $f: E \rightarrow E^{\prime}$ is a sequence of maps $\left(f_{n}\right)_{n=n_{0}}^{\infty}, f_{n} \in \operatorname{Mor}\left(E_{n}, E_{n}^{\prime}\right), n_{0} \geq \max \left\{n(E), n\left(E^{\prime}\right)\right\}$, such that the diagrams

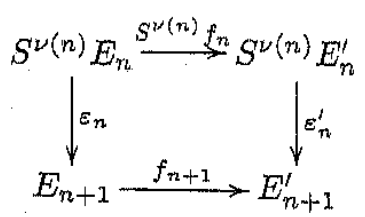

are homotopy commutative for all $n \geq n_{0}$.

We define the category of $\nu$-spectra $\mathcal{E}(\nu)$ as follows: the class of objects $\mathcal{O} \mathcal{E}(\nu)$ of $\mathcal{E}(\nu)$ consists of the spectra defined as above, and for each $E, E^{\prime} \in$ $\mathcal{O E}(\nu), \mathcal{M E}(\nu)\left(E, E^{\prime}\right)$ is the set of all maps of spectra from $E$ into $E^{\prime}$.
Definition 3.3. Two maps of spectra $f, f^{\prime}: E \rightarrow E^{\prime}$ are homotopic if there is $n_{1} \in \mathbb{N}$ such that $f_{n}$ is homotopic to $f_{n}^{\prime}$ whenever $n \geq n_{1}$.

DEFINITION 3.4. We say that $f \in \mathcal{M E}(\nu)\left(E, E^{\prime}\right)$ is a homotopy equivalence if there exists $g \in \mathcal{M E}(\nu)\left(E^{\prime}, E\right)$ such that $g \circ f \in \mathcal{M E}(\nu)(E, E)$ is homotopic to $\operatorname{id}_{E}$ and $f \circ g \in \mathcal{M E}(\nu)\left(E^{\prime}, E^{\prime}\right)$ is homotopic to $\operatorname{id}_{E^{\prime}}$.

DefinITION 3.5. Two spectra $E$ and $E^{\prime}$ are said to be homotopy equivalent or to have the same homotopy type if there is a homotopy equivalence $f \in \mathcal{M E}(\nu)\left(E, E^{\prime}\right)$. The homotopy type of the spectrum $E$ is denoted by $[E]$.

R.EMARK 3.6. For a given spectrum $E=\left(\left(E_{n}\right)_{n=n(E)}^{\infty},\left(\varepsilon_{n}\right)_{n=n(E)}^{\infty}\right)$ its homotopy type is uniquely determined by the homotopy type of the pointed space $E_{n}$ with $n$ sufficiently large. In particular, if $\left(\varepsilon_{n}\right)_{n=n(E)}^{\infty}$ is replaced by another sequence $\left(\varepsilon_{n}^{\prime}\right)_{n=n(E)}^{\infty}$ satisfying conditions (b) and (c) of Definition 3.1 then the resulting spectrum has the same homotopy type as the original one. Therefore, in order to define a homotopy type [E] one only needs a sequence of spaces $E=\left(E_{n}\right)_{n=n(E)}^{\infty}$ such that $S^{\nu(n)} E_{n}$ is homotopy equivalent to $E_{r_{k}+1}$ for $n$ sufficiently large.

Denote by $\underline{0}$ a spectrum such that for each $n \geq 0$, the space $E_{n}$ consists only of a base point, and $\varepsilon_{n}$ maps the point in $E_{n}$ to the point in $E_{n+1}$. The suspension functor acts trivially on such spaces, i.e. $S E_{n}=E_{n}$, and hence $\underline{Q} \in \mathcal{O} \mathcal{E}(\nu)$ for every $\nu: \mathbb{N} \rightarrow \mathbb{N}$.

DEFINITION 3.7. We say that the homotopy type of a spectrum $E$ is trivial if $E$ is homotopy equivalent to $\underline{0}$.

4. The Conley index. In this section we keep all the assumptions and notations of Section 2. In addition, we denote by $P_{n}: H \rightarrow H$ the orthogonal projection onto $H^{n}:=\bigoplus_{i=0}^{n} H_{i}$. Let $H_{n}^{-}$(resp. $H_{n}^{+}$), $n \geq 1$, denote the $L$-invariant subspace of $H_{n}$ corresponding to the part of the spectrum of $L$ with negative (resp. positive) real part. Define $\nu: \mathbb{N} \rightarrow \mathbb{N}$ by $\nu(n):=\operatorname{dim} H_{n+1}^{-1}, n=0,1, \ldots$

We begin with a special case. Assume $f: H \rightarrow H, f(x)=L x+K(x)$, is an $\mathcal{L S}$-vector field satisfying condition (2.3), i.e. $f$ is subquadratic. Let $\eta: \mathbb{R} \times H \rightarrow H$ be the $\mathcal{L} S$-flow generated by $f$ and let $X \subset H$ be an isolating neighbourhood for $\eta$.

Define $f_{n}: H^{n} \rightarrow H^{n}$ and $F_{n}: H^{n+1} \times[0,1] \rightarrow H^{n+1}$ by

$f_{n}(x):=L x+P_{n}(K(x)), \quad F_{n}(x, s):=L x+(1-s) P_{n}(K(x))+s P_{n+1}(K(x))$. Let $\eta_{n}: \mathbb{R} \times H^{n} \rightarrow H^{n}$ denote the flow induced by $f_{n}$, and $\xi_{n}: \mathbb{R} \times H^{n+1} \times$ $[0,1] \rightarrow H^{n+1.1}$ the family of flows induced by $F_{n}$. 
We will need the following.

LEMMA 4.1. There exists $n_{0} \in \mathbb{N}$ such that if $n \geq n_{0}$ then $X_{n}:=X \cap H^{n}$ is an isolating neighbourhood for the flow $\eta_{n}$ and for the family of flows $\xi_{n-1}$.

Proof. Define a family of $\mathcal{L S}$-vector fields, $F: H \times[0,1] \rightarrow H$, by

$$
\begin{aligned}
F(x, s):= & L x+(1+n)(1-n s) P_{n+1}(K(x)) \\
& +n[(n+1) s-1] P_{n}(K(x)) \text { for } \frac{1}{n+1}<s \leq \frac{1}{n}, \\
F(x, 0):= & f(x) .
\end{aligned}
$$

Obviously for any $s \in[0,1]$ the $\mathcal{L S}$-vector field $F(\cdot, s)$ satisfies $(2.3)$. Thus $F$ generates a family of $\mathcal{L} \mathcal{S}$-flows $\xi: \mathbb{R} \times H \times[0,1] \rightarrow H$. Proposition 2.3 implies that $\operatorname{Inv}(X \times[0,1], \xi)$ is a compact subset of $X \times[0,1]$. Since $\operatorname{Inv}(X \times[0,1], \xi) \cap X \times\{0\} \subset \operatorname{Int}(X)$ there is $s_{0}>0$ such that

$$
\operatorname{Inv}(X \times[0,1], \xi) \cap X \times\left[0, s_{0}\right] \subset \operatorname{Int}(X) \times\left[0, s_{0}\right] .
$$

Choosing $n_{0}$ such that $1 / n_{0}<s_{0}$ we conclude the proof.

By Lemma 4.1, $X_{n}$ is an isolating neighbourhood for the flow $\eta_{n}, n \geq n_{0}$. Set $S_{n}:=\operatorname{Inv}\left(X_{n}, \eta_{n}\right)$. Thus $S_{n}$ admits an index pair $\left(Y_{n}, Z_{n}\right)$ (cf. [5], [16]) and the Conley index of $S_{n}$ is the homotopy type of the pointed space $Y_{n} / Z_{n}$. Let

$$
\begin{gathered}
D_{n}^{+}:=\left\{x \in H_{n}^{+}:\|x\| \leq 1\right\}, \quad D_{n}^{-}:=\left\{x \in H_{n}^{-}:\|x\| \leq 1\right\}, \\
\partial D_{n}^{+}:=\left\{x \in H_{n}^{+}:\|x\|=1\right\} .
\end{gathered}
$$

Let $S_{n+1, n}:=\operatorname{Inv}\left(X_{n+1} \times[0,1], \xi_{n}\right)$ and $S_{n+1, n}(s):=\left\{x \in X_{n+1}:(x, s) \in\right.$ $\left.S_{n+1, n}\right\}$. Clearly

$$
\left(Y_{n} \times D_{n+1}^{+} \times D_{n+1}^{-}, Z_{n} \times \partial D_{n+1}^{+} \times D_{n+1}^{-}\right)
$$

is an index pair for the isolated invariant set $S_{n}=S_{n+1, n}(0)$ with respect to the flow $\xi_{n}(\cdot, \cdot, 0)$. Thus the Conley index of $S_{n}$ with respect to $\xi_{n}(\cdot, \cdot, 0)$ is the homotopy type of

$$
\left(Y_{n} \times D_{n+1}^{+} \times D_{n+1}^{-}\right) /\left(Z_{n} \times \partial D_{n+1}^{+} \times D_{n+1}^{-}\right),
$$

which in turn is equal to the homotopy type of $S^{\nu(n)}\left(Y_{n} / Z_{n}\right)$. Moreover $X_{n+1}$ is an isolating neighbourhood for the flow $\xi_{n}(\cdot, \cdot, s)$ for all $s \in[0,1]$ Therefore, by the continuation property of the Conley index (see [5], [16]) $S^{\nu(n)}\left(Y_{n} / Z_{n}\right)$ is homotopy equivalent to $Y_{n+1} / Z_{n+1}$. Thus, in view of Remark 3.6, the sequence

$$
\left(E_{n}\right)_{n=n_{0}}^{\infty}:=\left(Y_{n} / Z_{n}\right)_{n=n_{0}}^{\infty}
$$

determines uniquely the homotopy type $[E]$.
DEFINITION 4.2. Let $\eta$ be an $\mathcal{L S}$-flow generated by a subquadratic $\mathcal{L S}$ vector field and let $X$ be an isolating neighbourhood for $\eta$. Define

$$
h_{\mathcal{L S}}(X, \eta):=[E] .
$$

We call $h_{\mathcal{L S}}(X, \eta)$ the $\mathcal{L S}$-homotopy Conley index of $X$ with respect to $\eta$ or simply the $\mathcal{L} \mathcal{S}$-homotopy index.

Turning to the general case assume that $f: H \rightarrow H, f(x)=L x+K(x)$, is an $\mathcal{L S}$-vector field and $X \subset H$ is an isolating neighbourhood for the local flow $\eta$ generated by $f$. Choose $s \in \mathbb{R}$ such that $X \subset B(0, s)$ and define $\mu: \mathbb{R} \rightarrow \mathbb{R}$ by

$$
\mu(t)= \begin{cases}1 & \text { if } t \leq s \\ 1+s-t & \text { if } s<t \leq s+1 \\ 0 & \text { if } t \geq s+1\end{cases}
$$

and $d: H \rightarrow[0,1]$ by $d(x)=\mu(\|x\|)$.

Clearly, the map $K_{1}: H \rightarrow H, K_{1}(x):=d(x) K(x)$, is completely continuous, locally Lipschitz continuous and the closure of $K_{1}(H)$ is compact. Hence $f_{1}: H \rightarrow H, f_{1}(x)=L x+K_{1}(x)$, is a subquadratic $\mathcal{L} \mathcal{S}$-vector field. Moreover, the flow $\eta_{1}$ generated by $f_{1}$ and the local flow $\eta$ coincide on $X$. That is, $\eta(x, t) \in X$ for $t \in[0, a]$ implies $\eta_{1}(x, t)=\eta(x, t)$ for $t \in[0, a]$. In particular, $X$ is an isolating neighbourhood for $\eta_{1}$. Note also that if $\eta_{2}$ is another $\mathcal{L S}$-flow generated by a subquadratic $\mathcal{L S}$-vector field $f_{2}$ such that $f_{2}(x)=f(x)$ for all $x \in X$ then

$$
h_{\mathcal{L S}}\left(X, \eta_{1}\right)=h_{\mathcal{L S}}\left(X, \eta_{2}\right)
$$

DEFinition 4.3. Let $f$ be an $\mathcal{L S}$-vector field, $\eta$ the local flow generated by $f$ and let $X$ be an isolating neighbourhood for $\eta$. Define

$$
h_{\mathcal{L S}}(X, \eta):=h_{\mathcal{L S}}\left(X, \eta_{1}\right)
$$

We call $h_{\mathcal{L S}}(X, \eta)$ the $\mathcal{L} \mathcal{S}$-homotopy Conley index of $X$ with respect to $\eta$ or simply the $\mathcal{L} S$-homotopy index. index.

The following propositions give the basic properties of the $\mathcal{L S}$-homotopy

Proposrtion 4.4 (Nontriviality). Let $\eta: D(\eta) \rightarrow H$ be the local flow generated by an $\mathcal{L} \mathcal{S}$-vector field and let $X \subset H$ be an isolating neighbourhood for $\eta$. If $h_{\mathcal{L S}}(X, \eta) \neq 0$ then $\operatorname{Inv}(X, \eta) \neq \emptyset$.

Proof. With no loss of generality we may suppose that $\eta$ is generated by a subquadratic $\mathcal{L S}$-vector field $f$. Let $F$ be the family of $\mathcal{L S}$-vector fields defined for $f$ in the proof of Lemma 4.1, so that $f=F(\cdot, 0)$. The family of $\mathcal{L} \mathcal{S}$-flows generated by $F$ will be denoted by $\xi$. Proposition 2.3 implies that $\operatorname{Inv}(X \times[0,1], \xi)$ is a compact subset of $X \times[0,1]$. Assume that $\operatorname{Inv}(X, \eta)=\emptyset$. Then there is $s_{0}>0$ such that 


$$
\operatorname{Inv}(X \times[0,1], \xi) \cap X \times\left[0, s_{0}\right]=\emptyset
$$

and therefore $\operatorname{Inv}\left(X_{n}, \eta_{n}\right)=\emptyset$ for each $n \geq 1 / s_{0}$. Now, the classical Conley index theory says that all the spaces $E_{n}, n \geq 1 / s_{0}$, obtained in our construction of a spectrum for $X$ have the homotopy type of one-point space and therefore $[E]=[0]$.

Proposition 4.5 (Continuation). Let $\Lambda$ be a compact, connected and locally contractible metric space. Assume that $\eta: D(\eta) \rightarrow H$ is the family of local flows generated by a family of $\mathcal{L S}$-vector fields $f: H \times \Lambda \rightarrow H$. Let $X$ be an isolating neighbourhood for the flow $\eta_{\lambda}$ for some $\lambda \in \Lambda$. Then there is a compact neighbourhood $C \subset \Lambda$ of $\lambda(\lambda \in \operatorname{Int}(C))$ such that

$$
h_{\mathcal{L S}}\left(X, \eta_{\mu}\right)=h_{\mathcal{L S}}\left(X, \eta_{\nu}\right) \quad \text { for all } \mu, \nu \in C .
$$

Proof. This is a direct consequence of the continuation property of the classical Conley index.

5. Applications to Hamiltonian systems. In this section we discuss some examples in which our theory is applied. These may show why the theory presented here can sometimes give better results than other Morse type theories recently developed.

Now, we briefly recall a general setting in which our examples will be discussed. For more details we refer the reader to [17]. Given a Hamiltonian $G \in C^{2}\left(\mathbb{R}^{2 m} \times \mathbb{R}, \mathbb{R}\right)$ which is $2 \pi$-periodic in $t$ consider the Hamiltonian system of differential equations

$$
\dot{z}=J \nabla G(z, t)
$$

where $J=\left[\begin{array}{cc}0 & -I \\ I & 0\end{array}\right]$ is the standard symplectic matrix. Here and throughout the rest of this section $\nabla$ denotes the gradient with respect to $z \in \mathbb{R}^{2 m}$. Throughout the section we assume

(5.2) $\|\nabla G(z, t)\| \leq c_{1}+c_{2}\|z\|^{s} \quad$ for $(z, t) \in \mathbb{R}^{2 m} \times \mathbb{R}$ and some $s>0$.

We will be concerned with the existence of $2 \pi$-periodic solutions of (5.1). Denote by $H=H^{1 / 2}\left(S^{1}, \mathbb{R}^{2 m}\right)$ the Hilbert space of $2 \pi$-periodic, $\mathbb{R}^{2 m}$-valued functions

$$
z(t)=a_{0}+\sum_{k=1}^{\infty}\left(a_{k} \cos (k t)+b_{k} \sin (k t)\right) \quad \text { where } a_{0}, a_{k}, b_{k} \in \mathbb{R}^{2 m}
$$

with the inner product given by

$$
\left\langle z, z^{\prime}\right\rangle_{H}=2 \pi\left\langle a_{0}, a_{0}^{\prime}\right\rangle+\pi \sum_{k=1}^{\infty} k\left\langle\left\langle a_{k}, a_{k}^{\prime}\right\rangle+\left\langle b_{k}, b_{k}^{\prime}\right\rangle\right)
$$

where $\langle a, b\rangle$ denotes the standard inner product of $a, b \in \mathbb{R}^{2 m}$.
Any $z \in H$ can also be written in the form

$$
z=\sum_{i=1}^{2 m} z_{i} e_{i}
$$

where $z_{i} \in H^{1 / 2}\left(S^{1}, \mathbb{R}\right)$ and $e_{1}, \ldots, e_{2 m}$ is the standard basis in $\mathbb{R}^{2 m}$.

It is well known that $z(t)$ is a $2 \pi$-periodic solution of (5.1) if and only if it is a critical point of the functional $\Phi \in C^{1}(H, \mathbb{R})$ defined by

$$
\Phi(z)=-\frac{1}{2}\langle L z, z\rangle_{H}-\phi(z),
$$

where

$$
\langle L z, z\rangle_{H}:=\int_{0}^{2 \pi}\langle J \dot{z}, z\rangle d t, \quad \phi(z):=\int_{0}^{2 \pi} G(z, t) d t
$$

(cf. [13]). It is also shown in [13]. that the mapping $\nabla \phi$ is compact and therefore $-\nabla \Phi: H \rightarrow H$ is a vector field which can be written in the form

$$
-\nabla \Phi(z)=L z+K(z)
$$

where $K:=\nabla \phi: H \rightarrow H$ is completely continuous.

Let

$$
\begin{aligned}
& H(0):=\operatorname{span}\left\{e_{1}, \ldots, e_{2 m}\right\}, \\
& H(k):=\operatorname{span}\left\{c_{k} e_{i}+s_{k} J e_{i}: i=1, \ldots, 2 m\right\}, \quad k \in \mathbb{Z}-\{0\},
\end{aligned}
$$

where $c_{k}(t):=\cos (k t)$ and $s_{k}(t):=\sin (k t)$. It follows from (5.3) and (5.5) that $L$ is a selfadjoint operator in $H$ such that $L z=0$ if $z \in H(0)$ and $-L z=$ $\pm z$ for every $z \in H( \pm k), k>0$. Set $H_{0}=H(0)$ and $H_{k}=H(k) \oplus H(-k)$, $k=1,2, \ldots$ Obviously, $H=\bigoplus_{k=0}^{\infty} H_{k}$ and $H_{0}=\operatorname{ker} L$. Each $z \in H$ can be written $z=\sum_{k=0}^{\infty} z_{k}$, where $z_{k}:=p_{k}(z)$ and $p_{k}: H \rightarrow H$ is the orthogonal projection onto $H_{k}, k=0,1, \ldots$

Using this notation we define $\Lambda: H \rightarrow H$ by $\Lambda(z):=z_{0}+\sum_{k=1}^{\infty} \frac{1}{k} z_{k}$.

Directly from the definition of the inner product in $H$ we have

$$
\nabla \phi(z)=\Lambda(w), \text { where } w(t)=\nabla G(z(t), t) .
$$

Thus we conclude that $F:=-\nabla \Phi$ is an $\mathcal{L S}$-vector field. Moreover, the map $\nu: \mathbb{N} \rightarrow \mathbb{N}$ which appears in the definition of spectrum is constant: $\nu(n)=2 m$ for each $k \in \mathbb{N}$.

Assume now that $A: \mathbb{R}^{2 m} \rightarrow \mathbb{R}^{2 m}$ is a linear symmetric map and consider the linear Hamiltonian system

$$
\dot{z}=J A z,
$$

In this case $G(z)=\frac{1}{2}\langle A z, z\rangle$, the $\mathcal{L S}$-vector field $-\nabla \Phi: H \rightarrow H$ corresponding to that system preserves all spaces $H_{k}$ and the restriction of $\nabla \Phi$ to $H_{k}, k \geq 1$, with respect to the basis $\left\{c_{k} e_{1}, \ldots, c_{k} e_{2 m}, s_{k} e_{1}, \ldots, s_{k} e_{2 m}\right\}$, 
is represented by the matrix

$$
T_{k}(A)=\left[\begin{array}{cc}
-\frac{1}{k} A & -J \\
J & -\frac{1}{k} A
\end{array}\right] ;
$$

moreover, $T_{0}(A)=-A$ on $H_{0}$ (see [17]).

The following numbers are well defined (cf. [1]):

$$
\begin{aligned}
i^{-}(A) & :=M^{-}(-A)+\sum_{k=1}^{\infty}\left(M^{-}\left(T_{k}(A)\right)-2 m\right), \\
i^{0}(A) & :=M^{0}(-A)+\sum_{k=1}^{\infty} M^{0}\left(T_{k}(A)\right),
\end{aligned}
$$

where $M^{-}(B)$ is the number (with multiplicity) of negative eigenvalues of a. symmetric matrix $B$ and $M^{0}(B)$ is the dimension of its kernel.

If $i^{0}(A)=0$ then $\nabla \Phi$ is a linear isomorphism. Then $S:=\{0\}$ is an isolated invariant set for the flow $\eta$ induced by $-\nabla \Phi$. Moreover, if $r>0$ then $D(r):=\{z \in H:\|z\| \leq r\}$ is an isolating neighbourhood and

$$
h_{\mathcal{L S}}(D(r), \eta)=[E]
$$

where $E$ is a spectrum such that $E_{n}=S^{p(n)}$ with $p(n)=i^{-}(A)+n \cdot 2 m$ for sufficiently large $n$

Thus, using elementary properties of $h_{\mathcal{L S}}$ we obtain the following two observations:

REMARK 5.1. Assume that $G(z, t)=\frac{1}{2}\left\langle A_{0} z, z\right\rangle+g(z, t)$, where $A_{0}$ is lin ear symmetric and $\nabla g(z, t)=o(|z|))$ uniformly in $t$ as $z \rightarrow 0$. If $i^{0}\left(A_{0}\right)=0$ then for $r$ sufficiently small and positive, $D(r):=\{z \in H:\|z\| \leq r\}$ is an isolating neighbourhood of $S=\{0\}$ and

where $E_{n}=S^{p(n)}$ for $n$ sufficiently large.

$$
h_{\mathcal{L S}}(D(r), \eta)=[E]
$$

REMARK 5.2. Assume that $G(z, t)=\frac{1}{2}\left\langle A_{\infty} z, z\right\rangle+g(z, t)$, where $A_{\infty}$ is linear symmetric and $\nabla g(z, t)$ is bounded. If $i^{0}\left(A_{\infty}\right)=0$ then for $r$ sufficiently large $D(r)$ is an isolating neighbourhood and

where $E_{n}=S^{p(n)}$ for $n$ sufficiently large.

$$
h_{\mathcal{L S}}(D(r), \eta)=[E]
$$

We begin our discussion with the following two lemmas.

LEMMA 5.3. Let $B$ be a Banach space and $F: B \times \mathbb{R}^{n} \ni(x, y) \mapsto$ $F(x, y) \in B$ be a $C^{p}$ map. Suppose that $F(0,0)=0, D_{B} F(0,0): B \rightarrow B$ is an isomorphism and the partial derivatives $D_{\mathbb{R}^{n}}^{i} F(0,0): \mathbb{R}^{n} \times \ldots \times \mathbb{R}^{n} \rightarrow B$ are zero for $i=1, \ldots, p$. Let $\phi: \mathbb{R}^{n} \supset V \ni y \mapsto \phi(y) \in B$ be the implicit function defined by $F(\phi(y), y)=0$, where $V$ is an open neighbourhood of
$0 \in \mathbb{R}^{n}$. Then the derivatives $D^{i} \phi(0): \mathbb{R}^{n} \times \ldots \times \mathbb{R}^{n} \rightarrow B$ are zero maps for $i=1, \ldots, p$.

LEMMA 5.4. Let $F: B \times \mathbb{R}^{n} \rightarrow B \times \mathbb{R}^{n}$ be a $C^{p+1}$-map, $F(x, y)=$ $\left(F_{1}(x, y), F_{2}(x, y)\right)$. Assume that $F_{1}: B \times \mathbb{R}^{n} \rightarrow B$ satisfies the assumptions of Lemma 5.3 and let $\phi: \mathbb{R}^{n} \supset V \ni y \mapsto \phi(y) \in B$ be the implicit function defined by $F_{1}(\phi(y), y)=0, y \in V$. Additionally, suppose that $D_{B} F_{2}(0,0)$ : $B \rightarrow \mathbb{R}^{n}$ is zero. Define $\psi: \mathbb{R}^{n} \supset V \ni y \mapsto \psi(y) \in \mathbb{R}^{n}$ by $\psi(y)=F_{2}(\phi(y), y)$. Then $D^{i} \psi(0)=D_{\mathbb{R}^{n}}^{i} F_{2}(0,0): \mathbb{R}^{n} \times \ldots \times \mathbb{R}^{n} \rightarrow \mathbb{R}^{n}$ for $i=1, \ldots, p+1$.

As an immediate consequence of the above lemmas we obtain

COROLlary 5.5. Let $F$ be as in Lemma 5.4. Assume that the first nonvanishing derivative $D_{\mathbb{R}^{n}}^{i} F_{2}(0,0): \mathbb{R}^{n} \times \ldots \times \mathbb{R}^{n} \rightarrow \mathbb{R}^{n}$ is nondegenerate, i.e. $D_{\mathbb{R}^{\text {r }}}^{i} F_{2}(0,0)(y, \ldots, y)=0$ iff $y=0$. Then the origin $(0,0) \in B \times \mathbb{R}^{n}$ is isolated in the set $F^{-1}(0)$ of zeros of $F: B \times \mathbb{R}^{n} \rightarrow B \times \mathbb{R}^{n}$.

Now, we turn to our examples.

EXAMPLE 5.1. Let $m=1$ and define $G: \mathbb{R}^{2} \times \mathbb{R} \rightarrow \mathbb{R}$ by

$$
G(x, y, t)=\frac{1}{2}\left(x^{2}+y^{2}\right)+\left(x^{3}-3 x y^{2}\right) \cos (3 t),
$$

i.e. $G(z, t)=\frac{1}{2}\left\langle A_{0} z, z\right\rangle+g(z, t)$, where $z=(x, y) \in \mathbb{R}^{2}, A_{0}=\operatorname{Id}$ and $g(z, t)=\left(x^{3}-3 x y^{2}\right) \cos (3 t)$.

Let $\mathcal{A}_{0}$ denote the derivative of $-\nabla \Phi$ at 0 . Evidently it is a selfadjoint operator and ker $\mathcal{A}_{0}=H(1) \subset H_{1}$ is a subspace of dimension 2 spanned by $u_{1}, u_{2}$, where

$$
u_{1}(t)=\cos (t) \cdot e_{1}+\sin (t) \cdot e_{2}, \quad u_{2}(t)=-\sin (t) \cdot e_{1}+\cos (t) \cdot e_{2} .
$$

The subspaces $V_{1}=\operatorname{im} \mathcal{A}_{0}$ and $V_{2}=\operatorname{ker} \mathcal{A}_{0}$ are orthogonal in $H$ and $H=$ $V_{1} \oplus V_{2}$. Let $\pi_{1}, \pi_{2}: H \rightarrow H$ denote the orthogonal projections onto $V_{1}, V_{2}$, respectively. Define $F_{1}: V_{1} \oplus V_{2} \rightarrow V_{1}$ by $F_{1}\left(v_{1}, v_{2}\right)=-\pi_{1} \circ \nabla \Phi\left(v_{1}, v_{2}\right)$ and $F_{2}: V_{1} \oplus V_{2} \rightarrow V_{2}$ by $F_{2}\left(v_{1}, v_{2}\right)=-\pi_{2} \circ \nabla \Phi\left(v_{1}, v_{2}\right)$. Clearly, $D_{V_{1}} F_{1}(0,0)=$ $\pi_{1} \circ \mathcal{A}_{0 \mid V_{1}}: V_{1} \rightarrow V_{1}$ is an isomorphism and $D_{V_{2}} F_{1}(0,0)=\pi_{1} \circ \mathcal{A}_{0 \mid V_{2}}:$ $V_{2} \rightarrow V_{1}$ is zero. By Lemma 5.3 the implicit function $\phi: U \rightarrow V_{1}$ defined by $F_{1}\left(\phi\left(v_{2}\right), v_{2}\right)=0, U$ an open neighbourhood of the origin in $V_{2}$, satisfies $D \phi(0)=0$. Consequently, by Lemma $5.4, D^{i} \psi(0)=D_{V_{2}}^{i} F_{2}(0,0)$ for $i=1,2$, where $\psi\left(v_{2}\right)=F_{2}\left(\phi\left(v_{2}\right), v_{2}\right)$.

Choosing the basis $\left\{u_{1}(t), u_{2}(t)\right\}$ we introduce a coordinate system in $V_{2}$. For each $v_{2} \in V_{2}$ one has

$$
\begin{aligned}
v_{2}(t) & =a u_{1}(t)+b u_{2}(t) \\
& =(a \cos (t)-b \sin (t)) e_{1}+(a \sin (t)+b \cos (t)) e_{2}, \quad a, b \in \mathbb{R} .
\end{aligned}
$$

We replace $x$ by $a \cos (t)-b \sin (t)$ and $y$ by $a \sin (t)+b \cos (t)$ in $\nabla G$. The resulting map $u(t)$ is an element of $H^{1 / 2}\left(S^{1}, \mathbb{R}^{2}\right)$ and

$$
\pi_{2}(u(t))=\left\langle u(t), u_{1}(t)\right\rangle_{H} \cdot u_{1}(t)+\left\langle u(t), u_{2}(t)\right\rangle_{H} \cdot u_{2}(t) .
$$


Using the above procedure one shows that up to a positive factor,

$$
F_{2}(0,(a, b))=\left(a^{2}-b^{2},-2 a b\right)
$$

in some neighbourhood of $0 \in V_{2}$. Thus $D_{V_{2}} F_{2}(0,0)=0$ and $D_{V_{2}}^{2} F_{2}(0,0)$ is nondegenerate at 0 . Therefore, by Corollary 5.5, $0 \in H$ is isolated in $\nabla \Phi^{-1}(0)$. Since we are dealing with a gradient vector field, $0 \in H$ is an isolated invariant set for the semiflow generated by $F=-\nabla \Phi$.

Now, if the local flow on $V_{2}$ is generated by $F_{2}(0, \cdot): U \rightarrow V_{2}$ then we easily compute the Conley index of $\{0\}$ which is equal to $\left[S^{1} \vee S^{1}, *\right]$ the homotopy type of the join of 2 copies of 1-dimensional pointed spheres. Using (5.8) we then find that the Conley index of $\{0\}$ in $H$ is equal to tho homotopy type of the spectrum $E$ with $E_{n}=S^{2 n+3} \vee S^{2 n+13}$, the wedge of two pointed spheres of dimension $2 n+3, n=1,2, \ldots$

ExAmple 5.2. Let $m=1$ and define $G: \mathbb{R}^{2} \times \mathbb{R} \rightarrow \mathbb{R}$ by

$$
G(x, y, t)=\frac{1}{2}\left(x^{2}+y^{2}\right)+\left(x^{2}+y^{2}\right)^{2}+h(x, y, t) .
$$

The same arguments as in Example 5.1 show that $0 \in H$ is an isolated invariant set for the semiflow $\eta$ generated by $F=-\nabla \Phi$, where $\Phi: H \rightarrow \mathbb{R}$ is the functional corresponding to the system (5.1) with $G$ as above. This time we find that up to a positive factor,

$$
F_{2}(0,(a, b))=\left(a^{3}+a b^{2}, a^{2} b+b^{3}\right)
$$

in a neighbourhood of $0 \in V_{2}$.

Now, the Conley index of $\{0\}$ is the homotopy type of the spectrum $E$ with $E_{n}=S^{2 n+2}, n=1,2, \ldots$

Throughout the rest of this section we will be concerned with a Hamiltonian $G \in C^{2}\left(\mathbb{R}^{2} \times \mathbb{R}, \mathbb{R}\right) 2 \pi$-periodic in $t$ and satisfying the following two conditions:

(G.0) there exists $c_{0}>0$ such that $|z|<c_{0}$ implies $G(z, t)=\frac{1}{2}\left\langle A_{0} z, z\right\rangle+$ $g_{0}(z, t)$, where $A_{0}$ is a linear symmetric operator and $\nabla g_{0}(z, t)=$ $o(|z|)$ uniformly in $t$ as $z \rightarrow 0$;

(G. $\infty)$ there exists $c_{\infty}>0$ such that $|z|>c_{\infty}$ implies $G(z, t)=\frac{1}{2}\left\langle A_{\infty} z, z\right\rangle$ $+g_{\infty}(z, t)$, where $A_{\infty}$ is linear symmetric, $i^{0}(A)=0$ and $\nabla g_{\infty}$ is bounded.

If $G$ satisfies the above assumptions then 0 is a critical point of $\nabla \Phi$. Generalizing some earlier results, Szulkin has proved in [17] (Theorem 7.2) that if

$$
i^{-}\left(A_{\infty}\right) \notin\left[i^{-}\left(A_{0}\right), i^{0}\left(A_{0}\right)+i^{-}\left(A_{0}\right)\right]
$$

then (5.1) has a nontrivial solution (in addition to the trivial one $z=0$ ).

The following examples show how the $\mathcal{L S}$-Conley index can be used to obtain similar results even if (5.9) is not satisfied.
EXAMPLE 5.3. Suppose that

(i) $G(x, y, t)=\frac{1}{2}\left(x^{2}+y^{2}\right)+\left(x^{3}-3 x y^{2}\right) \cos (3 t)$ if $x^{2}+y^{2}<c_{1}$, for some $c_{1}>0$

(ii) $G(x, y, t)=\frac{1}{2} d\left(x^{2}+y^{2}\right)+g(x, y, t)$ if $x^{2}+y^{2}>c_{2}$ for some $c_{2}>c_{1}$ with $g$ having a bounded derivative and $d \in(0,1) \cup(1,2)$.

From Example 5.1 we know that $S=\{0\}$ is an isolated invariant set for the $\mathcal{L S}$-flow generated by $-\nabla \Phi$ whose Conley index is the homotopy type of a spectrum $E$ with $E_{n}=S^{2 n+3} \vee S^{2 n+3}$.

It is also evident that if we take $U$ to be an open ball in $H$ of a sufficiently large radius, then $U$ is an isolating neighbourhood. Let $S_{\infty}$ denote the maximal invariant subset contained in $U$. The Conley index of $S_{\infty}$ is the homotopy type of a spectrum $E^{\prime}$ such that:

(a) $E_{n}^{\prime}=S^{2 n+2}$ if $d \in(0,1)$

(b) $E_{n}^{\prime}=S^{2 n+4}$ if $d \in(1,2)$,

for $n$ sufficienty large.

Since $[E] \neq\left[E^{\prime}\right]$ we conclude that $S_{\infty} \neq\{0\}$ and therefore there must be another zero of $\nabla \Phi$, which gives a nontrivial solution of (5.1).

Finally, one easily verifies that $i^{-}\left(A_{0}\right)=2, i^{0}\left(A_{0}\right)=2, i^{-}\left(A_{\infty}\right)=2$ if $d \in(0,1)$ and $i^{-}\left(A_{\infty}\right)=4$ if $d \in(1,2)$. This shows that (5.9) is not satisfied.

However, our example has also a disadvantage. Consider the linear isomorphism $\widetilde{L}: H \rightarrow H$ defined

$$
\widetilde{L} z= \begin{cases}L z & \text { if } z \in \bigoplus_{k=1}^{\infty} H_{k}, \\ z & \text { if } z \in H_{0}\end{cases}
$$

Let $U \subset H$ be an open and bounded set and let $f: \bar{U} \rightarrow H$ be of the form $f(z)=\widetilde{L}(z)+K(z)$, where $K: \vec{U} \rightarrow H$ is completely continuous and $f$ has no zeros on the boundary of $\bar{U}$. Then one defines the Leray-Schauder degree with respect to $\widetilde{L}$ to be

$$
\operatorname{deg}(f, U, 0):=\operatorname{deg}_{\mathrm{LS}}\left(\operatorname{Id}+\widetilde{L}^{-1} \circ K, U, 0\right)
$$

where the right hand side is the classical Leray-Schauder degree (cf. [8]). Now, an easy computation shows that in our example the local degree of

$$
-\nabla \Phi(z)=L z+K(z)=\widetilde{L} z+\widetilde{K}(z)
$$

at 0 is equal to -2 and if $U$ is a disc centred at 0 with radius sufficiently large then $\operatorname{deg}(-\nabla \Phi, U, 0)$ is 1 . Consequently, there is at least one $z \neq 0$ such that $\nabla \Phi(z)=0$.

In the next example that kind of argument cannot be used. 


\section{EXAMPLE 5.4. Suppose that}

(i) $G(x, y, t)=\frac{1}{2}\left(x^{2}+y^{2}\right)+\left(x^{2}+y^{2}\right)^{2}+h(x, y, t)$ if $x^{2}+y^{2} \leq c_{1}$ for some $c_{1}>0$, with $h$ being a smooth perturbation of order higher than 4 (with respect to $x$ and $y$ variables);

(ii) $G(x, y, t)=\frac{1}{2} d\left(x^{2}+y^{2}\right)+g(x, y, t)$ if $x^{2}+y^{2} \geq c_{2}$ for some $c_{2}>c_{1}$ with $g$ having a bounded derivative and $d \in(1,2)$.

From Example 5.2 we know that $S=\{0\}$ is an isolated invariant subset for the $\mathcal{L S}$-flow generated by $-\nabla \Phi$ whose Conley index is the homotopy type of a spectrum $E$ with $E_{n}=S^{2 n+2}$.

Again it is clear that an open ball $U \subset H$ of a sufficiently large radius is an isolating neighbourhood. The Conley index of $S_{\infty}=\operatorname{Inv}(\bar{U})$ is the homotopy type of a spectrum $E^{\prime}$ such that $E_{n}^{\prime}=S^{2 n+4}$ for $n$ sufficiently large. We have $[E] \neq\left[E^{\prime}\right]$ and therefore $S_{\infty} \neq\{0\}$. Since $F$ is a gradient vector field, $S_{\infty}$ has to have at least two stationary points.

Note that in this example we have $i^{-}\left(A_{0}\right)=2, i^{0}\left(A_{0}\right)=2, i^{-}\left(A_{\infty}\right)=4$ which gives $i^{-}\left(A_{\infty}\right) \in\left[i^{-}\left(A_{0}\right), i^{-}\left(A_{0}\right)+i^{0}\left(A_{0}\right)\right]$ and thus the theorem. proved e.g. in [1], [9], [10], [17] cannot be applied. The Leray-Schauder degree gives us no extra information either. The local degree at 0 and the degree on a sufficiently large disc of $-\nabla \Phi$ (with respect to $\widetilde{L}$ ) are equal to 1 .

\section{References}

[1] H. Amann and E. Zehnder, Periodic solutions of asymptotically linear Hamilto nian systems, Manuscripta Math. 32 (1980), 149-189.

[2] V. Benci, A new approach to the Morse-Conley theory and some applications, Ann. Mat. Pura Appl. (4) 158 (1991), 231-305.

[3] K. C. Chang, Infinite Dimensional Morse Theory and Multiple Solution Problems, Bîrkhäuser, Boston, 1993.

4] K. C. Chang, S. P. Wu and S. J. Li, Multiple periodic solutions for an asymptotically linear wave equation, Indiana Univ. Math. J. 31 (1982), 721-731.

[5] C. C. Conley, Isolated Invariant Sets and the Morse Index, CBMS Regional Conf. Ser. in Math. 38, Amer. Math. Soc., Providence, R.I., 1978.

[6] C. C. Conley and E. Zehnder, Morse type index theory for flows and periodic solutions for Hamiltonian equations, Comm. Pure Appl. Math. 37 (1984), 207-253.

[7] K. Deimling, Ordinary Differential Equations in Banach Spaces, Lecture Notes in Math. 596, Springer, 1977

[8] J. Dugundji and A. Granas, Fixed Point Theory, Vol. I, Monograf. Mat, 61 PWN-Polish Sci. Publ., 1982.

[9] S. Li and J. Q. Liu, Morse theory and asymptotic linear Hamiltonian systems, J. Differential Equations 79 (1988), 53-73.

[10] Y. Long, The Index Theory of Hamiltonian Systems with Applications, Science Press, Beijing, 1993.

[11] J. Mawhin and M. Willem, Critical Point Theory and Hamiltonian Systems, Springer, 1989.
[12] K. Mischaikow, Conley index theory, in: Dynamical Systems, R. Johnson (ed.) Lecture Notes in Math. 1609, Springer, 1995, 119-207.

13] P. H. Rabinowitz, Minimax Methods in Critical Point Theory with Application to Differential Equations, CBMS Regional Conf. Ser. in Math. 35, Amer. Math. Soc. Providence, R.I., 1986.

[14] -, Free vibrations for a semilinear wave equation, Comm. Pure Appl. Math. 31 $(1978), 31-68$

[15] K. Rybakowski, The Homotopy Index and Partial Differential Equations, Universitext, Springer, 1987.

[16] D. Salamon, Connected simple systems and the Conley index of isolated invariant sets, Trans. Amer. Math. Soc. 291 (1985), 1-41.

[17] A. Szulkin, Cohomology and Morse theory for strongly indefinite functionals, Math. Z. 209 (1992), 375-418.

18] - Index theories for indefinite functionals and applications, in: Topological and Variational Methods for Nonlinear Boundary Value Problems (Cholin, 1995), Pitman Res. Notes Math. Ser. 365, Longman, 1997, 89-121.

[19] C. Troestler and M. Willem, Nontrivial solution of a semilinear Schrödinger equation, Comm. Partial Differential Equations 21 (1996), 1431-1449.

[20] G. W. Whitehead, Recent Advances in Homotopy Theory, CMBS Regional Conf Ser. in Math. 5, Amer. Math. Soc., Providence, R.I., 1970.

Institute of Mathematics

Polish Academy of Science

18 Abrahama. St.

81-825 Sopot, Poland

E-mail: kgeba@ksinet.univ.gda.pl

izydorek@mifgate.pg.gda.pl

amp@ksinet.univ.gda.pl 